\title{
Depression in secondary epilepsy: relation to lesion laterality
}

\author{
Mario F Mendez, Jody L Taylor, Robert C Doss, Patricia Salguero
}

St Paul-Ramsey

Medical Center, University of

Minnesota, St Paul, MN, USA

M F Mendez

J L Taylor

R C Doss

$P$ Salguero

Correspondence to:

Dr M F Mendez

Department of Neurology,

t Paul-Ramsey Medical

Center, St Paul, MN 55101,

Received 30 April 1993

and in revised form

12 July 1993.

Accepted 21 July 1993

Table Patients with epilepsy who had depression *

\begin{tabular}{lllll}
\hline $\begin{array}{l}\text { Patient } \\
\text { No }\end{array}$ & Age/Sex & Seizuret & Lesion aetiologył & \\
\hline 1 & $44 \mathrm{M}$ & SPS,GTCS & Temporal AVM & LateralityS \\
2 & $62 \mathrm{~F}$ & CPS,GTCS & Parietal infarct & L \\
3 & $49 \mathrm{~F}$ & CPS,GTCS & Frontotemporal contusions & B \\
4 & $56 \mathrm{M}$ & CPS & Temporal glioma & L \\
5 & $37 \mathrm{M}$ & GTCS & Temporal contusions & B \\
6 & $51 \mathrm{~F}$ & GTCS & Frontal-caudate infarcts & B \\
7 & $84 \mathrm{~F}$ & CPS & Frontal infarct & L \\
8 & $31 \mathrm{~F}$ & GTCS & Hemisphere hypoplasia & L \\
9 & $40 \mathrm{~F}$ & SPS & Frontal contusions & B \\
10 & $43 \mathrm{M}$ & GTCS & Sylvian fissure AVM & R \\
11 & $59 \mathrm{M}$ & GTCS & Frontotemporal contusions & B \\
12 & $54 \mathrm{~F}$ & CPS,GTCS & Frontal infarct & L \\
13 & $31 \mathrm{~F}$ & CPS,GTCS & Frontal abscess & L \\
14 & $30 \mathrm{~F}$ & CPS,GTCS & Temporal hemangioma & L \\
15 & $32 \mathrm{M}$ & CPS,GTCS & Temporal contusion & L \\
16 & $33 \mathrm{~F}$ & GTCS & Frontal contusion & L \\
17 & $28 \mathrm{M}$ & CPS & Hemisphere atrophy & R \\
18 & $36 \mathrm{~F}$ & CPS & Frontotemporal infarct & L \\
19 & $40 \mathrm{~F}$ & GTCS & Multiple infarcts & B \\
20 & $21 \mathrm{M}$ & CPS & Occipital wound & L \\
21 & $27 \mathrm{M}$ & SPS & Frontotemporal contusions & B \\
22 & $67 \mathrm{~F}$ & GTCS & Temporal AVM & L \\
23 & $21 \mathrm{M}$ & GTCS & Hydrocephalus & B \\
24 & $70 \mathrm{~F}$ & GTCS & Temporal infarct & L \\
25 & $67 \mathrm{~F}$ & GTCS & Parietal infarct & L \\
\hline
\end{tabular}

${ }^{\star}$ DSM-III-R diagnoses were major depression in Nos 1-7, bipolar disorder, depressed in No 8-11, and depressive disorder not otherwise specified in nos 12-25. +CPS = Complex partial seizures; SPS = Simple partial seizures; GTCS = generalised tonic-clonic seizures. $\ddagger \mathrm{AVM}=$ Arteriovenous malformation. $\$ \mathrm{~L}=$ left; $\mathrm{R}=$ right; $\mathrm{B}=$ bilateral.

\section{$(\Im$ Neurol Neurosurg Psychiatry 1994;57:232-233)}

Depression is common in patients with epilepsy. ${ }^{1}$ Among such patients, depressive disorders are the most frequent reason for

\begin{abstract}
Patients with epilepsy often have depres-
Abstract
Patients with epilepsy often have depressive disorders. This association may be particularly prominent in secondary epilepsy from a left hemisphere lesion. Among 1611 outpatients with epilepsy 272 patients were identified whose seizures originated from a structural brain lesion other than mesial temporal sclerosis. Sustained depressive disorders had occurred in 25 (9\%) of these patients with secondary epilepsy. The depressed patients were compared with the remaining patients without depression with regard to location of lesion laterality and seizure variables. The only group difference was unilateral left hemisphere lesions in $58 \%$ of the patients with depression compared with only $21 \%$ of the non-depressed patients $\left(\chi^{2}=10 \cdot 4\right.$, $p=0.006)$. This finding supports the idea of a relation of depression with epileptogenic lesions in the left hemisphere.
\end{abstract}

admission to hospitals for psychiatric illness and the main reason for taking psychotropic drugs. $^{2-4}$ Although depression can be ictally related, most is interictal without a direct relation with seizures. ${ }^{35}$ The greater frequency of interictal depression among patients with epilepsy than among comparably disabled patients suggests a biological cause for depression rather than a psychosocial reaction to having a seizure disorder. ${ }^{367}$

Based on EEG localisation, interictal depression may occur more often in those with epileptogenic foci in the left hemisphere. ${ }^{38}$ Localisation by EEG can be misleading, and identification of a causative structural lesion for epilepsy is a more accurate method of localisation. This study focused on patients with epilepsy and with verifiable lesions known to be the source of their seizures. The study evaluated these patients for depressive disorders, lesion laterality, and location, and seizure variables.

\section{Methods}

Among all patients with epilepsy who attended a neurology clinic from 1984-92, those with a lesion on CT or MRI were identified. Patients with MRI findings suggestive of mesial temporal sclerosis were excluded because of reservations about the presence of this change in many patients. Patients with definite structural lesions determined to be the source of secondary epilepsy were included in the study. These patients were assessed for seizure type, auras, well lateralised EEG foci, and the use of barbiturate anticonvulsant drugs that could cause depression.

Among these patients with secondary epilepsy, we identified those with a history of interictal depressive disorders (excluding brief reactive depressions) previously diagnosed by a psychiatrist. All depressive disorders met DSM-III-R ${ }^{9}$ criteria for a sustained depressive illness not considered by the psychiatrist to be a direct consequence of psychosocial stressors or neurological disability.

\section{Results}

Out of 1611 outpatients in our epilepsy clinic, there were 272 patients with neuroimaged lesions, and a depressive disorder occurred in 25 (9.2\%; table). This "depression group" (15 women, 10 men; $44 \cdot 7$ 
[SD 16.8]) years old; all right handed) did not differ from the remaining "non-depression group" (111 women, 136 men; 42.2 $(15 \cdot 7)$ years old) in sex and age.

The depression group had a significantly higher percentage of patients with left hemispheric lesions than the non-depression group $\left(14(58 \%) v 63(21 \%) ; \chi^{2}=10 \cdot 4, \mathrm{p}=0.006\right)$. There were no differences in right hemisphere lesions (3 (12\%) $v 69(28 \%))$ or in intrahemispheric localisation. Also, there were no significant differences between depression $v$ non-depression groups in secondarily generalised tonic-clonic seizures (18 (72\%) v $205(83 \%)$ ) complex partial seizures $(10(40 \%)$ v $72(29 \%))$, auras $(7(28 \%) v 51$ (21\%)), left temporal EEG foci $(8(32 \%) v$ $51(21 \%))$, right temporal EEG foci (5 (20\%) $v 50(20 \%))$, or the presence of barbiturate therapy $(3(12 \%) \quad v 25(10 \%))$. Finally, although seizure frequency tended to be decreased during depressive episodes, the reported frequencies were not temporally accurate enough to be analysed separately.

\section{Discussion}

Several studies maintain that patients with epileptiform discharges lateralised to the left hemisphere have a specific predisposition to interictal depression. ${ }^{3810}$ Our findings are consistent with this hypothesis and extend this observation to patients with secondary epilepsy, with lateralised structural lesions.

Depression is common among patients with epilepsy. Studies have shown up to a $75 \%$ incidence of depression in such patients, ${ }^{11}$ and heightened depressive traits in patients with epilepsy compared with normal controls. ${ }^{1710}$ Although patients with epilepsy experience feelings of loss of control, stigmatisation, and other psychosocial difficulties, investigators report greater interictal depression among patients with seizures than among those with similar chronic disabilities. ${ }^{367}$ Furthermore, interictal depression is more common among patients whose seizures have a focal rather than a primarily generalised onset. ${ }^{112}$ These findings suggest that interictal depression is often biological rather than due to the psychosocial difficulties of having a seizure disorder.

Both a biological depression and epilepsy could result from a common hypometabolic lesion in the left hemisphere. Strokes and other hypometabolic brain lesions are especially likely to precipitate depression when they occur in the left hemisphere. ${ }^{13}$ Positron emission tomography studies show that even small, imperceptible epileptogenic foci are surrounded by extensive areas of "surround inhibition," 14 and this interictal hypometabolism may be even more prominent in those with depression. ${ }^{15}$ Moreover, hypometabolism in areas of the left hemisphere may characterise primary depressive disorders. ${ }^{16}$

We conclude that interictal depression in epilepsy can be an organic mental disorder related to the underlying lesion in the left hemisphere. Consistent with the laterality of emotions suggested by gelastic seizures in the left hemisphere and dyscrastic seizures in the right hemisphere, ${ }^{17}$ depression could follow relative right hemisphere prominence from left hemisphere hypometabolism. Future studies could explore this interhemispheric "thymic imbalance" theory as a cause for depression in epilepsy.

1 Robertson MM. Depression in patients with epilepsy: an overview. Semin Neurol 1991;11:182-9.

2 Betts TA. Epilepsy and the mental hospital. In: Reynolds EH, Trimble MR, eds. Epilepsy and psychiatry. New York: Churchill Livingstone, 1981:175-84.

3 Mendez MF, Cummings JL, Benson DF. Depression in epilepsy. Significance and phenomenology. Arch Neurol 1986;43:766-70.

4 Wilensky AJ, Leal KW, Dudley DL, Friel PN. Characteristics of psychotropic drug use in an epilepsy center population. Epilepsia 1981;22:247.

5 Blumer D. Epilepsy and disorders of mood. In: Smith DB, Treiman DG, Trimble MR, eds. Neurobehavioral problems in epilepsy. New York: Raven Press, 1991: problems

6 Kogeorgos J, Fonagy P, Scott DF. Psychiatric symptom patterns of chronic epileptics attending a neurologica clinic: A controlled investigation. Br $\mathcal{F}$ Psychiatry 1982 140:236- 43

7 Standage KF, Fenton GW. Psychiatric symptom profiles of patients with epilepsy: a controlled investigation Psychol Med 1975;5:152-60.

8 Altshuler LL, Devinsky O, Post RM, Theodore W Depression, anxiety, and temporal lobe epilepsy. Laterality of focus and symptoms. Arch Neurol 1990, 47:284-8.

9 American Psychiatric Association. Diagnostic and statistical manual III-revised. Washington, DC: APA Press, 1987.

10 Bear D, Fedio P. Quantitative analysis of interictal behavior in temporal lobe epilepsy. Arch Neurol 1977;34: 454-67.

11 Roy A. Some determinants of affective symptoms in epileptics. Can ₹ Psychiatry 1979;24:554-6.

12 Currie S, Heathfield KWG, Henson RA, Scott DF Clinical course and prognosis of temporal lobe epilepsy-a survey of 666 patients. Brain 1971;92. 173-90.

13 Robinson RG, Szetela B. Mood change following left hemisphere brain injury. Ann Neurol 1981;9:447-53.

14 Engel J Jr, Kuhl DE, Phelps ME, Mazziotta JC. Interictal cerebral glucose metabolism in partial epilepsy and its relation to EEG changes. Ann Neurol 1982;12:510-7.

15 Bromfield EB, Altshuler L, Leiderman DB, et al. Cerebral metabolism and depression in patients with complex partial seizures. Arch Neurol 1992;49:617-23.

16 Baxter LR, Schwartz JM, Phelps ME, et al. Reduction of prefrontal glucose metabolism common to three types of depression. Arch Gen Psychiatry 1989;46:243-50.

17 Sackeim HA, Greenberg MS, Weiman AL, et al. Hemispheric asymmetry in the expression of positive and negative emotions. Neurologic evidence. Arch Neurol 1982;39:210-8. 